\title{
Modified Kocher-Langenbeck approach for the treatment of posterior wall or column acetabular fractures: The one-incision two-window method
}

\section{Tien-Yu Yang}

Chiayi Chang Gung Memorial Hospital https://orcid.org/0000-0003-4685-0310

Kuo-Chin Huang ( $\nabla$ kc2672@gmail.com )

https://orcid.org/0000-0002-3225-1504

\section{Technical advance}

Keywords: one-incision two-window method, posterior wall acetabular fracture, posterio column acetabular fracture, modified Kocher-Langenbeck approach

Posted Date: November 3rd, 2020

DOl: https://doi.org/10.21203/rs.3.rs-58691/v3

License: (c) (i) This work is licensed under a Creative Commons Attribution 4.0 International License.

Read Full License 


\section{BMC Musculoskeletal Disorders}

\section{Modified Kocher-Langenbeckapproach for the treatment of posterior wall or column acetabular fractures: The one-incision two-window method

\author{
--Manuscript Draft--
}

Manuscript Number:

Full Title:

Modified Kocher-Langenbeck approach for the treatment of posterior wall or column acetabular fractures: The one-incision two-window method

Article Type:

Original Article

Keywords:

one-incision two-window method, posterior wall acetabular fracture, posterior column acetabular fracture, modified Kocher-Langenbeck approach

Corresponding Author: Kuo-Chin Huang, MD, PhD

Corresponding Author Secondary Information:

Corresponding Author's Institution:

Corresponding Author's Secondary Institution:

First Author:

Tien-Yu Yang, MD

First Author Secondary Information:

Order of Authors:

Tien-Yu Yang, MD

Po-Yao Chuang, MD

Tsan-Wen Huang, MD

Kuo-Chin Huang, MD, PhD

Order of Authors Secondary Information:

Manuscript Region of Origin:

TAIWAN 
STROBE Statement - Checklist of items that should be included in reports of cohort studies

\begin{tabular}{|c|c|c|c|}
\hline & $\begin{array}{c}\text { Item } \\
\text { No }\end{array}$ & Recommendation & $\begin{array}{c}\text { Page } \\
\text { No }\end{array}$ \\
\hline Title and abstract & 1 & $\begin{array}{l}\text { (a) Indicate the study's design with a commonly used term in the title or the } \\
\text { abstract } \\
\text { (b) Provide in the abstract an informative and balanced summary of what was } \\
\text { done and what was found }\end{array}$ & $1-2$ \\
\hline \multicolumn{4}{|l|}{ Introduction } \\
\hline Background/rationale & 2 & $\begin{array}{l}\text { Explain the scientific background and rationale for the investigation being } \\
\text { reported }\end{array}$ & 3 \\
\hline Objectives & 3 & State specific objectives, including any prespecified hypotheses & 4 \\
\hline \multicolumn{4}{|l|}{ Methods } \\
\hline Study design & 4 & Present key elements of study design early in the paper & 6 \\
\hline Setting & 5 & $\begin{array}{l}\text { Describe the setting, locations, and relevant dates, including periods of } \\
\text { recruitment, exposure, follow-up, and data collection }\end{array}$ & 6 \\
\hline Participants & 6 & $\begin{array}{l}\text { (a) Give the eligibility criteria, and the sources and methods of selection of } \\
\text { participants. Describe methods of follow-up } \\
\text { (b) For matched studies, give matching criteria and number of exposed and } \\
\text { unexposed }\end{array}$ & 6 \\
\hline Variables & 7 & $\begin{array}{l}\text { Clearly define all outcomes, exposures, predictors, potential confounders, and } \\
\text { effect modifiers. Give diagnostic criteria, if applicable }\end{array}$ & $6-7$ \\
\hline $\begin{array}{l}\text { Data sources/ } \\
\text { measurement }\end{array}$ & $8^{*}$ & $\begin{array}{l}\text { For each variable of interest, give sources of data and details of methods of } \\
\text { assessment (measurement). Describe comparability of assessment methods if } \\
\text { there is more than one group }\end{array}$ & $6-7$ \\
\hline Bias & 9 & Describe any efforts to address potential sources of bias & 7 \\
\hline Study size & 10 & Explain how the study size was arrived at & 7 \\
\hline Quantitative variables & 11 & $\begin{array}{l}\text { Explain how quantitative variables were handled in the analyses. If applicable, } \\
\text { describe which groupings were chosen and why }\end{array}$ & 7 \\
\hline Statistical methods & 12 & $\begin{array}{l}\text { (a) Describe all statistical methods, including those used to control for } \\
\text { confounding } \\
\text { (b) Describe any methods used to examine subgroups and interactions } \\
\text { (c) Explain how missing data were addressed } \\
\text { (d) If applicable, explain how loss to follow-up was addressed } \\
\text { (e) Describe any sensitivity analyses }\end{array}$ & 7 \\
\hline \multicolumn{4}{|l|}{ Results } \\
\hline Participants & $13 *$ & $\begin{array}{l}\text { (a) Report numbers of individuals at each stage of study—eg numbers potentially } \\
\text { eligible, examined for eligibility, confirmed eligible, included in the study, } \\
\text { completing follow-up, and analysed } \\
\text { (b) Give reasons for non-participation at each stage } \\
\text { (c) Consider use of a flow diagram }\end{array}$ & 8 \\
\hline Descriptive data & $14 *$ & $\begin{array}{l}\text { (a) Give characteristics of study participants (eg demographic, clinical, social) } \\
\text { and information on exposures and potential confounders } \\
\text { (b) Indicate number of participants with missing data for each variable of interest } \\
\text { (c) Summarise follow-up time (eg, average and total amount) }\end{array}$ & 8 \\
\hline Outcome data & $15 *$ & Report numbers of outcome events or summary measures over time & $8-9$ \\
\hline
\end{tabular}




\begin{tabular}{|c|c|c|c|}
\hline \multicolumn{3}{|c|}{$\begin{array}{l}\text { Main results } 16 \text { (a) Give unadjusted estimates and, if applicable, confounder-adjusted estimates and their precision } \\
\text { (eg, } 95 \% \text { confidence interval). Make clear which confounders were adjusted for and why } \\
\text { they were included } \\
\text { (b) Report category boundaries when continuous variables were categorized } \\
\text { (c) If relevant, consider translating estimates of relative risk into absolute risk for a } \\
\text { meaningful time period }\end{array}$} & $8-10$ \\
\hline Other analyses & 17 & $\begin{array}{l}\text { Report other analyses done — eg analyses of subgroups and interactions, and sensitivity } \\
\text { analyses }\end{array}$ & $8-10$ \\
\hline \multicolumn{4}{|l|}{ Discussion } \\
\hline Key results & & Summarise key results with reference to study objectives & 11 \\
\hline Limitations & 19 & $\begin{array}{l}\text { Discuss limitations of the study, taking into account sources of potential bias or imprecision. } \\
\text { Discuss both direction and magnitude of any potential bias }\end{array}$ & 14 \\
\hline Interpretation & 20 & $\begin{array}{l}\text { Give a cautious overall interpretation of results considering objectives, limitations, } \\
\text { multiplicity of analyses, results from similar studies, and other relevant evidence }\end{array}$ & $\begin{array}{l}11- \\
14\end{array}$ \\
\hline Generalisability & 21 & Discuss the generalisability (external validity) of the study results & 14 \\
\hline \multicolumn{4}{|c|}{ Other information } \\
\hline Funding & & $\begin{array}{l}\text { Give the source of funding and the role of the funders for the present study and,if } \\
\text { applicable, for the original study on which the present article is based }\end{array}$ & 15 \\
\hline
\end{tabular}

*Give information separately for exposed and unexposed groups.

Note: An Explanation and Elaboration article discusses each checklist item and gives methodological background and published examples of transparent reporting. The STROBE checklist is best used in conjunction with this article (freely available on the Web sites of PLoS Medicine at http://www.plosmedicine.org/, Annals of Internal Medicine at http://www.annals.org/, and Epidemiology at http://www.epidem.com/). Information on the STROBE Initiative is available at http://www.strobe-statement.org. 


\section{Modified Kocher-Langenbeck approach for the treatment of posterior wall or column acetabular fractures: The one-incision two-window method}

Running Title: Modified K-L Approach for Posterior Acetabular Fractures

Tien-Yu Yang, MD ${ }^{1,2}$; Po-Yao Chuang, MD ${ }^{1,2}$; Tsan-Wen Huang, $M D^{1,2}$;

Kuo-Chin Huang, MD, $\mathrm{PhD}^{1,2, *}$

1. Department of Orthopaedic Surgery, Chiayi Chang Gung Memorial Hospital, Chiayi, Taiwan

2. Chang Gung University College of Medicine, Taoyuan, Taiwan

E-mail addresses for all authors:

TYY, pku70@cgmh.org.tw

PYC, b9102072@cgmh.org.tw

TWH, b8601081@cgmh.org.tw

KCH, kc2672@gmail.com

* Corresponding author:

Kuo-Chin Huang, MD, PhD

Chiayi Chang Gung Memorial Hospital

No. 6, West Sec., Chia-Pu Rd., Pu-Tz City

Chiayi County 61363, Taiwan

Tel: +886-5-3621000 ext. 2855

Fax: +886-5-3623005

E-mail: kc2672@gmail.com 


\section{Competing interest}

The authors declare that they have no competing interests. They received no financial support for the research, authorship, and/or publication of this article. 


\section{Abstract}

2 Background: The Kocher-Langenbeck (K-L) approach is the standard method for the

3 treatment of posterior wall or column acetabular fractures. This approach allows direct

4 access to the posterior structures of the acetabulum, but is limited cranially and caudally by

5 the neurovascular bundle. The present study was conducted to assess the quality of

6 reduction and the incidence of complications in patients who underwent the modified

7 “one-incision two-window" K-L approach.

8 Design: Retrospective case series.

9 Setting: Single academic Level 1 trauma center.

10 Patients/Participants: Thirteen consecutive patients from 2015 to 2017 who sustained an

11 acute, displaced posterior wall or column acetabular fracture.

12 Intervention: Open reduction and internal fixation through the modified "one-incision

13 two-window" K-L approach.

14 Main Outcome Measurements: Radiographic reduction quality, incidence of complications,

15 visual analogue scale score, modified Harris hip score, and satisfaction rate.

16 Results: Among thirteen consecutive patients, seven patients were classified as having a

17 transverse with or without posterior wall fracture (group 1) and six patients had a solitary

18 posterior wall fracture (group 2). The mean operation time, mean intraoperative blood loss,

19 and mean incision wound length were estimated to be $103.8 \mathrm{~min}(60-120 \mathrm{~min}), 373.1 \mathrm{~mL}$

$20(100-700 \mathrm{~mL})$, and $9.7 \mathrm{~cm}(8.0-13.0 \mathrm{~cm})$, respectively. The radiographic quality of reduction

21 was graded as anatomical reduction (maximum residual displacement $[\mathrm{MRD}] \leq 2 \mathrm{~mm}$ ) in all 
22 cases, according to Matta's criteria. Concerning the incidence of complications, there were no

23 iatrogenic neurovascular injury, no surgical site infections, and no osteonecrosis of the

24 femoral head or heterotopic ossification in this cohort. One patient in group 1 experienced

25 deep vein thrombosis in the lesion leg. Another two patients in group 2 developed

26 posttraumatic osteoarthritis, with one diagnosed as Tonnis grade I lesion and the otheras

27 Tonnis grade III lesion. With respect to the clinical treatment outcome, the mean visual

28 analogue scale (VAS), mean modified Harris Hip Score (mHHS) and subjective satisfaction

28 rate were $1.7(1.0-2.0), 90.6(81-100)$, and $84.6 \%(80 \%-90 \%)$, respectively. Although there

29 was no significant difference in the satisfaction rate $(82.9 \%$ vs. $87.0 \%, P=0.941)$ at 12

30 months after surgery, group 1 patients had more increased VAS score (2.0 vs. 1.2, $P=0.016)$

31 and more decreased $\mathrm{mHHS}(87.7$ vs. 94.6, $P=0.014)$ than group 2 patients.

32 Conclusions: Our pilot study confirmed that the "one-incision two-window" K-L approach is

33 a simple, safe, reliable, and effective way to manage acute, displaced posterior wall or

34 column acetabular fractures.

35 Level of evidence: Therapeutic study, level IV.

36 Key Words: one-incision two-window method, posterior wall acetabular fracture, posterior

37 column acetabular fracture, modified Kocher-Langenbeck approach 


\section{Background}

2 According to the classification of Judet and Letournel, posterior wall acetabular

3 fracture is the most common type of elementary fracture pattern and transverse posterior

4 wall fracture is the most common associated fracture pattern. ${ }^{1,2}$ As in the case with other

5 displaced acetabular fracture types, the best treatment results are predicted on the basis of

6 anatomic fracture reduction and rigid fixation. ${ }^{1,3}$ Ordinarily, the Kocher-Langenbeck (K-L)

7 approach is the standard method for the treatment of posterior wall or column acetabular

8 fractures. ${ }^{4}$ This approach allows direct access to the outer surface of the posterior

9 structures and indirect access to the superior wall and quadrilateral surface; however, it is

10 limited cranially and caudally by the superior gluteal neurovascular bundle (SGB) and the

11 medial circumflex femoral artery (MCFA), respectively. ${ }^{4}$ Thus, various modifications of the

12 K-L approach had been reported with varying clinical results. ${ }^{5-8}$ Some techniques aimed at

13 sparing the division of the short external rotators (SERs), thus preventing iatrogenic damage

14 to the vascularity of the femoral head and of the fracture fragments. ${ }^{5,6}$ Others flip a slice of

15 the greater trochanter anteriorly with the attached gluteus medius and vastus lateralis

16 muscles to provide free access to the superior wall area and avoid damage to the hip

17 abductor muscles due to vigorous retraction. ${ }^{7,8}$ However, posttraumatic osteoarthritis

18 (PTOA), osteonecrosis of the femoral head (ONFH), heterotopic ossification (HO), osteotomy

19 nonunion, and neurovascular injury still tend to diminish the treatment outcome of 
20 acetabular fractures despite adequate surgical exposure of the operative field for good

21 fracture reduction and rigid fixation. ${ }^{9-11}$

22 The current study reviews our experience with a modification of the K-L approach in

23 the treatment of acute, displaced posterior wall or column acetabular fractures. The

24 modification involves a minimally invasive approach by which the neurovascular bundles are

25 well protected from undue traction or damage by avoiding vigorous retraction. The study

26 was conducted to assess the quality of reduction and the incidence of complications in

27 patients who underwent the modified "one-incision two-window" K-L surgical approach.

28 Surgical technique (Fig. 1,2,3)

29 The patient is placed in a lateral decubitus position with the affected side uppermost.

30 No skeletal traction is applied to the injured hip. In line with the longitudinal axis of the

31 femur, skin incision is started $5 \mathrm{~cm}$ distal to the posterior tip of greater trochanter (PTGT).

32 After passing the PTGT, the skin incision is continued $5 \mathrm{~cm}$ further in the direction of the

33 posterior superior iliac spine. The iliotibial tract is divided sharply in line with its fibers, and

34 the gluteus maximus muscle is split bluntly along the raphe, which indicates the inter-neurovascular interval between the SGB and the inferior gluteal neurovascular bundle for the upper one-third and lower two-third of the gluteus maximus muscle, respectively.

37 After removing the layer of fat and bursa covering the SERs, the insertions of the piriformis tendon and the conjoined tendon of the gemellus and obturator internus muscles are meticulously identified and divided $1 \mathrm{~cm}$ lateral from their femoral insertions to protect the 
40 MCFA, which helps supply blood to the neck and head of the femur. By retracting the

41 divided tendon stumps gently, the first window is created to expose the posterior wall,

42 ischial body, and ischial tuberosity. In this window, the displaced fracture fragments can be

43 reduced and fixed provisionally with Kirschner wires. The posterior column may be

44 stabilized first with a small reconstruction plate along the greater sciatic notch to hold the

45 reduction. Final plate fixation is then completed using a precontoured long pelvic

46 reconstruction plate that is slid under the gluteal muscles, in line with the posterior rim of

47 the acetabulum, and spans from the ischium to the superior wall area. After fixing the distal

48 end of the plate with two screws into its concave bend, another window is created through

49 the tensor fascia lata and gluteal muscles for exposure of the proximal screw holes of the

50 plate. With the assistance of finger palpation in hip abduction position, the proximal screws

51 can be applied accurately and safely. After definite fracture fixation and delicate soft tissue

52 debridement, the piriformis and conjoined tendons are reattached to their femoral stump

53 footprint by using 1-0 Vicryl suture. At the end of the surgical procedure, the wound is

54 closed by layers without placing a drain. Postoperatively, no prophylaxis (indomethacin or radiation) against $\mathrm{HO}$ is used. An intermediate-restrictive rehabilitation protocol is suggested, including immediate toe-touch weightbearing with progression to full weightbearing by 12 weeks. ${ }^{12}$ 


\section{Participating patients and study significance} and 3 women) were treated by a single surgeon (TTY) for acute, displaced posterior wall or column acetabular fractures by using the modified "one-incision two-window" K-L surgical approach. Among these patients, seven had transverse with or without posterior wall acetabular fracture and six had posterior wall acetabular fracture alone. At the time of study enrollment, we summarized the collected data, which included demographic characteristics, fracture pattern, surgery details, and radiographic and clinical outcome measurements, among others. These data were analyzed to study the effectiveness of the modified "one-incision two-window" K-L surgical approach in the treatment of acute, displaced posterior wall or column acetabular fractures. The modification involves a minimally invasive approach that is believed to provide a simple, safe, reliable, and effective way to manage these complex lesions.

Radiographic and clinical outcome measurements

Regular radiographic and clinical assessments were carried out immediately before and after surgery - that is, preoperatively; immediately after surgery; and at 1, 2, 3, 6, and 12 months postoperatively. Computed tomography scan of the pelvis with three-dimensional reconstruction was routinely performed preoperatively to helpconfirm the fracture types according to the classification of Judet and Letournel. ${ }^{1,2}$ The radiographic 
80 quality of fracture reduction was assessed by measuring the maximum residual

81 displacement (MRD) of the walls and columns on digitized, standard anteroposterior and

82 two oblique pelvic views. and then graded according to Matta's criteria, as follows:

83 anatomical (MRD $\leq 2 \mathrm{~mm}$ ), satisfactory (MRD between 2 and $3 \mathrm{~mm}$ ), and unsatisfactory

84 (MRD $\geq 3 \mathrm{~mm}$ ). ${ }^{13-16}$ PTOA was evaluated using the Tonnis classification modified by adding

85 quantitative measurements of joint space narrowing and joint congruence to differentiate

86 early lesions. ${ }^{17}$ Patients were clinically evaluated for abduction power according to the

87 Medical Research Council grading system, and for the presence of a lurch gait pattern or a

88 Trendelenburg sign. The overall clinical outcomes were assessed according to the visual

89 analog scale (VAS) score, modified Harris Hip Score (mHHS), and subjective satisfaction

90 rate. ${ }^{18}$ The mean follow-up period averaged 20.6 months (range, 12-36 months).

\section{Statistical analysis}

Between-group comparisons were performed using univariable analysis.

93 Mann-Whitney U-test and Fisher's exact test were used to analyze numerical and nominal

94 variables, respectively. Significance was set at $P<0.05$ (two-sided). SPSS 12.0 (SPSS Inc.,

95 Chicago, IL, USA) was used for all analyses.

\section{Ethics statement}

The data were analyzed after the approval by the ethics committee (institutional 
101 Results

102 Analyses of patient characteristics and related variables (Table 1)

103 In this 3-year-long study, we enrolled 13 patients who underwent the modified

104 "one-incision two-window" K-L surgical approach for the treatment of acute, displaced

105 posterior wall or column acetabular fractures. Among them, seven patients were classified

106 as having a transverse with or without posterior wall fracture (group 1) and six patients had

107 a solitary posterior wall fracture (group 2). The mean patient age and mean body mass

108 index were 43.5 years (24-67 years) and $26.1 \mathrm{~kg} / \mathrm{m}^{2}\left(20.8-38.0 \mathrm{~kg} / \mathrm{m}^{2}\right)$, respectively; 10

109 patients (76.9\%) were men and 3 patients (23.1\%) were women. One patient in group 1 had

110 ipsilateral sciatic nerve (SN) injury and presented with preoperative drop foot deformity.

111 The mean operation time, mean intraoperative blood loss, and mean incision wound length

112 were estimated to be $103.8 \mathrm{~min}(60-120 \mathrm{~min}), 373.1 \mathrm{~mL}(100-700 \mathrm{~mL})$, and $9.7 \mathrm{~cm}(8.0-13.0$

$113 \mathrm{~cm}$ ), respectively. Although there was a trend toward a longer operation time (111 min vs.

$11495 \mathrm{~min}, P=0.123)$ and more intraoperative blood loss $(442.9 \mathrm{~mL} v \mathrm{~s} .291 .7 \mathrm{~mL}, P=0.071)$ in

115 group1, between-group comparison revealed no significant differences in all these

116 demographic characteristics and related variables (all $P \geq 0.071$ ).

117 Analyses of the quality of reduction and the incidence of complications (Table 2)

118 With respect to the quality of fracture reduction, we measured the MRD of the walls

119 and columns on plain radiographs taken immediately after surgery. We found that none of 
120 the residual wall or column fracture displacements were $>2 \mathrm{~mm}$; thus, the radiographic

121 quality of reduction was graded as anatomical reduction (MRD $\leq 2 \mathrm{~mm}$ ) in all cases,

122 according to Matta's criteria. All fractures achieved union, and there was no loss of

123 reduction and fixation during the follow-up period. Concerning the incidence of

124 complications, there were no iatrogenic SN or SGB injury, no superficial or deep surgical site

125 infections (SSIs), and no ONFH or HO in this cohort. However, one patient in group 1

126 developed deep vein thrombosis (DVT) and two patients in group 2 showed PTOA on

127 follow-up radiographs. Because acetabular fracture surgery may confer a high risk for

128 bleeding with anticoagulation, the patient with DVT received conservative treatment

129 through the shared decision-making program. Until the date of the final follow-up, this

130 patient did not have proximal extension of thrombosis, pulmonary embolism (PE) event, or

131 recurrent DVT/PE. With respect to PTOA, one patient was found to have Tonnis grade I

132 lesion (4 months after surgery, asymptomatic and without progression) and another patient

133 was found to have Tonnis grade III lesion (6 months after surgery, symptomatic progression).

134 Because of failed conservative treatment, the patient with advanced PTOA underwent total

135 hip arthroplasty (THA) at 6 months after posterior wall acetabular fracture dislocation.

136 Analyses of clinical outcome measures at 12 months after surgery (Table 3)

137 After the exclusion of 1 patient who underwent THA because of Tonnis grade III PTOA,

13812 patients were included in the analyses of clinical outcome measures at 12 months after

139 surgery. The mean VAS score, mean $\mathrm{mHHS}$, and subjective satisfaction rate were 1.7 
140 (1.0-2.0), $90.6(81-100)$, and 84.6\% (80\%-90\%), respectively. Although there was no

141 significant difference in satisfaction rate (82.9\% vs. $87.0 \%, P=0.941)$, group 1 patients had

142 more increased VAS score (2.0 vs. 1.2, $P=0.016)$ and more decreased $\mathrm{mHHS}$ (87.7 vs. 94.6,

$143 P=0.014)$ than group 2 patients.

144144

145145 
Our pilot study revealed that the modified "one-incision two-window" K-L surgical

148 approach is a simple, safe, reliable, and effective way to manage acute, displaced posterior

149 wall or column acetabular fractures. The proposed approach used a curved skin incision

150 averaging $9.7 \mathrm{~cm}$ (range, 8-13 cm) in length and provided an adequate working space for

151 manipulation by the surgeon even in patients with severe obesity (SDC 1). Within the first

152 window, fractured lesions were well exposed, reduced, and fixed provisionally. The

153 precontoured long pelvic reconstruction plate for definite fracture fixation could also be

154 easily placed in line with the posterior rim of the acetabulum by sliding under thegluteal

155 muscles. Without vigorous retraction, distal and proximal plate screws could beaccurately

156 and safely inserted in direct view through window 1 and window 2 , respectively. With this

157 technique, our results showed that an anatomical reduction with MRD $\leq 2 \mathrm{~mm}$ was achieved

158 in all patients. Further, an excellent functional outcome was attained in our series, average,

159 as assessed using the mHHS system.

160 The aim of surgical treatment of displaced acetabular fractures is to establish a stable

161 anatomical reduction with a functional, mobile, and pain-free hip. ${ }^{13,14}$ The accuracy of

162 fracture reduction and internal fixation correlates strongly with functional outcomes. ${ }^{10,15}$

163 The conventional K-L approach is the standard method for posterior wall and column

164 acetabular fractures; however, access to the superior wall area is limited. ${ }^{7}$ By using this 

acetabular fracture types, and fair or poor clinical results as measured using the d'Aubigne

167 score in $30 \%$ of cases. ${ }^{1}$ In the series of Gansslen et al., the authors reported a similar result with a $76 \%$ anatomical reduction rate and only $56.7 \%$ of their patients had a functionally satisfactory result. ${ }^{19}$ Although Bogdan et al. reported a $100 \%$ anatomical reduction rate and

170 functionally good results, as many as $18 \%$ of their patients required additional anterior 171 approach to facilitate reduction and fixation. ${ }^{20}$ Some other surgeons used a trochanteric flip 172 osteotomy to improve visualization of the superior wall area of the acetabulum from the 173 conventional K-L exposure, and reported good radiographic and clinical results. ${ }^{7,8}$ Additional 174 bone and soft tissue procedures might allow better visualization, more accurate reduction, 175 and easier fixation of the cranial acetabular fragments; however, the more extensile the 176 approach used, the higher the rate of associated surgical risk and complications. For 177 example, trochanteric nonunion is believed to occur in $2 \%-15 \%$ of cases following 178 trochanteric osteotomy. ${ }^{21}$ Without adding a bone or soft tissue procedure to the conventional K-Lapproach, vigorous manipulations for visualization, reduction of the fractured fragments, and even screw fixation of a bridging plate may lead to substantial stretching and laceration of the surrounding soft tissues, which subsequently may contribute to iatrogenic neurovascular injuries, $\mathrm{HO}, \mathrm{ONFH}$, and SSIs, among others. ${ }^{22-24} \mathrm{~A} 2 \%$ rate of intervention-related nerve injury (IRNI) associated with acetabular fractures was reported in a retrospective study of prospectively collected data from the German Pelvic Trauma Registry. ${ }^{23}$ In that 12 -year-long 
study including a total of 2236 patients from 29 hospitals, Lehmann et al. found that the K-L approach was associated with the highest proportion of IRNIs. ${ }^{23}$ Apart from the SN, which is typically injured in posterior approaches, the inferior ramification of the deep SGB is considered at risk when the gluteal muscles is retracted $>3 \mathrm{~cm}$ above the greater trochanter. ${ }^{23-25}$ Likewise, vigorous retraction might increase soft tissue injury, lead to

191 necrotic muscle in the injury zone, and increase the incidence of $\mathrm{HO}$ and SSIs. ${ }^{26}$ Some

192 researchers have suggested a modification involving sparing the division of the SERs, and

193 declared that it could prevent iatrogenic damage to the MCFA and thus reduce the risk of secondary ONFH. ${ }^{5,6}$ However, there was no direct evidence supporting this hypothesis.

One was used for controlling the posterior structures by gently elevating the SER flap, which preserved the MCFA and protected the SN. The other was used for governing the superior wall area by splitting the gluteal muscles, which avoided vigorous retraction and prevented SGB injury, HO, or SSIs. Despite the promising radiographic and clinical results, we encountered one case of DVT (7.7\%) and two cases of PTOA (15.4\%) in the present study. Major trauma and surgery are major situational triggers for DVT/PE. ${ }^{27}$ Kinking of femoral vessels at the time of impact and an intrapelvic hematoma compressing the pelvic vasculature might contribute to thrombus formation in the lesion limb after injury to the posterior structures of the acetabulum. ${ }^{27-29}$ Although acetabular fracture surgery confer a 
206 should prevail throughout the clinical pathway in these high-risk patients. PTOA is still one

207 of the most common complications of acetabular fractures and usually requires THA

208 thereafter. ${ }^{30}$ Even if anatomical fracture reduction was achieved in the present study, the

209 possibility of PTOA development could not be excluded owing to the increased rate of

210 chondrocyte apoptosis in intra-articular acetabular fractures with or without hip

211 dislocation. ${ }^{22}$

212 This study has several limitations. First, this pilot study was a retrospective case series

213 study with a small number of patients. Additionally, there was no control group for

214 comparison to provide reliable evidence for the superiority of the proposed technique.

215 Second, although the major fracture prototype was a posterior wall or column fracture,

216 several specific types of acetabular fractures were diagnosed in the current study. Thiscould

217 cause bias while analyzing the data, produces false conclusions, and is potentially

218 misleading. Prospectively randomized controlled trials are needed in the future to compare

219 this modified technique with the conventional approach for the specified fracture type.

220 Conclusion

221 This study introduced a modified K-L approach - the one-incision two-window method.

222 Through this technique, adequate working space was provided with good preservation of

223 the surrounding vital structures and without undue traction or damage secondary to

224 vigorous retraction. The fracture reduction and functional outcome were anatomical and

225 excellent, respectively. Further, there were no additional approach-related complications. 
226 Thus, we conclude that the proposed technique is a simple, safe, reliable, and effective way

227 to manage acute, displaced posterior wall or column acetabular fractures.

\section{Abbreviation}

229 K-L approach: Kocher-Langenbeck approach; MRD : maximum residual displacement;

230 VAS: visual analogue scale; mHHS: modified Harris Hip Score; SGB: superior gluteal

231 neurovascular bundle; MCFA: medial circumflex femoral artery; SERs: short external rotators

232 PTOA: posttraumatic osteoarthritis; ONFH: osteonecrosis of the femoral head;

233 HO: heterotopic ossification; PTGT: posterior tip of greater trochanter, TTY: Tien-Yu, Yang;

234 SN: sciatic nerve; SSI: surgical site infection; DVT: deep vein thrombosis; PE: pulmonary

235 embolism; THA: total hip arthroplasty; SDC: supplemental digital content; IRNI: intervention

236 related nerve injury.

\section{Declaration}

\section{Ethic approval and consent to participate}

239 The authors analyzed the datas after approval by the ethic committee (Institutional Review

240 Board) of the Chang Gung Memorial Hospital in Taiwan (Reference number: 201801360B0). We did

241 not obtain informed consent from the patient due to a statement of this committee, that analyzing

242 patient data retrospectively requires no informed consent. However, patient records and

243 information were anonymized and de-identified prior to analysis

\section{Consent for publication}

245 There is no any individual person's data in this manuscript.

246 Availability of data and materials

247 All data and information about materials are in the paper and/or available from the first author. 


\section{Competing interest}

249 The authors declare that they have no competing interests. They received no financial

250 support for the research, authorship, and/or publication of this article.

\section{$251 \quad$ Funding}

252 There is no funding from any institution or company in this study

\section{Authors Contribution}

254 TYY and $\mathrm{KCH}$ participated in the literature search, study design, data collection, data analysis

255 and interpretation, writing, and critical revision. PYC and TWH participated in the data

256 collection, data analysis and interpretation, writing, and critical revision.

\section{Acknowledgements}

258 We would like to thank Wen-Chi Liang for her assistance with data collection and statistical

259 analysis. 
261 1. Matta JM. Fractures of the acetabulum: accuracy of reduction and clinical results in

262 patients managed operatively within three weeks after the injury. J Bone Joint Surg Am.

$242 \quad$ 1996; 78(11): 1632-1645.

243 2. Porter SE, et al. Acetabular fracture patterns and their associated injuries. J Orthop

$244 \quad$ Trauma. 2008; 22(3): 165-170.

245 3. Moed BR, Carr SE, Watson JT. Open reduction and internal fixation of posterior wall

246 fractures of the acetabulum. Clin Orthop Relat Res. 2000; (377): 57-67.

247 4. Tosounidis TH, Giannoudis VP, Kanakaris NK, Giannoudis PV. The Kocher-Langenbeck

248 approach: state of the art. JBJS Essent Surg Tech. 2018; 8(2): e18.

249 5. Magu NK, Rohilla R, Arora S, More H. Modified Kocher-Lagenbeck approach for the

250 stabilization of posterior wall fractures of the acetabulum. J Orthop Trauma. 2011;

$251 \quad 25(4): 243-249$.

252 6. Josten C, Trabold O. Modified "2-portal" Kocher-Langenbeck approach: a

253 minimally-invasive procedure protecting the short external rotator muscles. J Orthop

$254 \quad$ Trauma. 2011; 25(4): 250-257.

255 7. Siebenrock KA, Gautier E, Ziran BH, Ganz R. Trochanteric flip osteotomy for cranial

256 extension and muscle protection in acetabular fracture fixation using a

257 Kocher-Langenbeck approach. J Orthop Trauma. 2006; 20(1 Suppl): S52-S56. 
258 8. Gupta S, Singh J, Virk JS. The role of trochanteric flip osteotomy in fixation of certain 259 acetabular fractures. Chin J Traumatol. 2017; 20(3): 161-165.

260 9. Roetman B, Seybold D, Keil D, Muhr G, Mollenhoff G. Long-term results after 261 acetabular fractures with respect to heterotopic ossifications. Zentrab/ Chir. 2006; 131(3): 188-193.

10. Briffa N, Pearce R, Hill AM, Bircher M. Outcomes of acetabular fracture fixation with ten years' follow-up. J Bone Joint Surg Br. 2011; 93(2): 229-236.

11. Studer P, Kosuge D, Rickman M, Solomom LB. Versatility of an extended posterior approach for the treatment of acetabular fractures with reference to the superior gluteal neurovascular bundle. J Orthop Trauma. 2016; 30(8): e289-e293.

12. Thompson SG, Phillip RD, Roberts A. How do orthopaedic surgeons and rehabilitation professionals interpret and assess toe touch weight bearing and partial weight bearing status in the rehabilitation setting? BMJ Open Sport Exerc Med. 2018; 4(1): e000326.

13. Matta JM, Anderson LM, Epstein HC, Hendricks P. Fractures of the acetabulum. A retrospective analysis. Clin Orthop Relat Res. 1986; (205): 230-240. prospective study. Clin Orthop Relat Res. 1986; (205): 241-250. indicators of outcome. Clin Orthop Relat Res. 2003; (407): 173-186. 
16. Shi HF, Xiong J, Chen YX, Wang JF, Wang YH. Radiographic analysis of the restoration of hip joint center following open reduction and internal fixation of acetabular fractures:a retrospective cohort study. BMC Musculoskelet Disord. 2014; 15: 277.

17. Kovalenko B, Bremjit P, Fernando N. Classification in brief: Tonnis classification of hip osteoarthritis. Clin Orthop Relat Res. 2018; 476(8): 1680-1684.

18. Frietman B, Biert J, Edwards MJR. Patient-reported outcome measures after surgery for an acetabular fracture. Bone Joint J. 2018; 100-B(5): 640-645.

19. Gansslen A, Hildebrand F, Kretek C. Transverse + posterior wall fractures of the acetabulum: epidemiology, operative management and long-term results. Acta Chir Orthop Traumatol. 2013; 80(1): 27-33.

20. Bogdan Y, Dwivedi S, Tornetta P 3rd. A surgical approach algorithm for transverse posterior all fractures aids in reduction quality. Clin Orthop Relat Res. 2014; 472(11):

21. Anglen JO, Hughes M. Trochanteric osteotomy for incarcerated hip dislocation due to $3338-3344$. interposed posterior wall fragments. Orthopaedics. 2004; 27(2): 213-216.

22. Petsatodis G, Antonarakos P, Chalidis B, Papadopoulos P, Christoforidis J, Pounaras J. Surgically treated acetabular fractures via a single posterior approach with a follow-up of 2-10 years. Injury. 2007; 38(3): 334-343.

23. Lehmann W, Hoffmann M, Fensky F, et al. What is the frequency of nerve injuries associated with acetabular fractures? Clin Orthop Relat Res. 2014; 472(11): 3395-3403. 
297 24. Solomon LB, Hofstaetter JG, Bolt MJ, Howie DW. An extended posterior approach to

298 the hip and pelvis for complex acetabular reconstruction that preserves the gluteal

299 muscles and their neurovascular supply. Bone Joint J. 2014; 96-B(1): 48-53.

300 25. Masonis JL, Bourne RB. Surgical approach, abductor function, and total hip arthroplasty

301 dislocation. Clin Orthop Relat Res. 2002; 405: 46-53.

302 26. Firoozabadi R, Alton T, Sagi HC. Heterotopic ossification in acetabular fracture surgery.

303 J Am Acad Orthop Surg. 2017; 25(2): 17-124.

304 27. Streiff MB, Agnelli G, Connors JM, et al. Guidance for the treatment of deep vein

305 thrombosis and pulmonary embolism. J Thromb Thrombolysis. 2016; 41(1): 32-67.

306 28. Montgomery KD, Geerts WH, Potter HG, Helfet DL. Thromboembolic complications in

307 patients with pelvic trauma. Clin Orthop Relat Res. 1996; 329: 68-87.

308 29. Panteli M, Lampropoulos A, Guthoff C, Giannoudis PV. Can we do better preventing

309 thromboembolic events following pelvic and acetabular injuries? Injury. 2013; 44(12):

$310 \quad 1673-1676$.

311 30. Moed BR. Improving results in posterior wall acetabular fracture surgery. J Trauma.

312 2007; 62(6 Suppl): S63.

313

314 


\section{$315 \quad$ Figure legends}

316 Figure 1. The modified "one-incision two-window" Kocher-Langenbeck surgical approach. (A)

317 Posterior structures of the right hip joint. (B) Window 1 (W1) is used for controlling the

318 posterior structures by gently elevating the short external rotators (SERs) as a flap, which

319 preserves the medial circumflex femoral artery (MCFA) and protects the sciatic nerve(SN).

320 After provisional reduction and fixation, a precontoured long pelvic reconstruction plate can

321 be slid under the gluteal muscles, in line with the posterior rim of the acetabulum, spanning

322 from the ischium to the superior wall area. Through this window, the distal end of the plate

323 is fixed with two screws into its concave bend. (C) Window 2 (W2) is used for governing the

324 superior wall area by splitting the gluteal muscles, which avoids vigorous retraction and

325 prevents superior gluteal neurovascular bundle (SGB) injury. Through this window, the

326 proximal plate screws can be applied accurately and safely in direct view.

327 Figure 2. The procedure of making the second window. (A) Applying the distal screws at

328 ischium through the first window. (B\&C) Anteriorly dissect the fascia lata upon the fascia

329 layer. (D) Incise the fascia lata at the proximal screw level, then split the gluteal muscle with

330 finger to the plate to make the second window.

331 Figure 3. Picture A represents the first and second windows. Picture B\&C represent the

332 proximal screws applying from the second window with verification by fluoroscopy.

\section{Supplemental digital content}

334 SDC 1. A 28-year-old male patient with a body mass index of $33.2 \mathrm{~kg} / \mathrm{m}^{2}$ suffered from left

335 posterior wall acetabular fracture and hip dislocation secondary to a motor vehicle accident. 
336 (A and B) Preoperative anteroposterior pelvic view and computed tomography scan of the

337 pelvis with three-dimensional reconstruction. (C and D) Postoperative oblique pelvic views.

338 (E) Photograph taken immediately after completing the procedure through the modified

339 "one-incision two-window" Kocher-Langenbeck approach. The length of the incision wound

340 was $9 \mathrm{~cm}$. 
Table 1. Demographic data of patients treated using the modified Kocher-Langenbeck approach.

\begin{tabular}{|c|c|c|c|c|}
\hline & $\begin{array}{c}\text { Total } \\
\text { (n=13) }\end{array}$ & $\begin{array}{l}\text { Group } 1 \\
(n=7)\end{array}$ & $\begin{array}{l}\text { Group } 2 \\
(n=6)\end{array}$ & $P$ value ${ }^{\S}$ \\
\hline Gender ( $n, \%)$ & & & & 1.000 \\
\hline Female & $3(23.1 \%)$ & $2(28.6 \%)$ & $1(16.7 \%)$ & \\
\hline Bone mass index $\left(\mathrm{kg} / \mathrm{m}^{2}\right)$ (mean, range) & $26.1(20.8-38.0)$ & $25.4(20.8-38.0)$ & $27.0(22.6-33.2)$ & 0.600 \\
\hline Preoperative sciatic nerve injury $(n, \%)$ & $1(7.7 \%)$ & $1(14.3 \%)$ & $0(0 \%)$ & 0.377 \\
\hline Incision wound length (cm) (mean, range) & $9.7(8-13)$ & $9.7(8-13)$ & $9.7(9-10)$ & 0.946 \\
\hline
\end{tabular}

Group1, transverse with or without posterior wall acetabular fracture; group 2, posterior wall acetabular fracture alone.

$\S P$ value, the calculated probability showing that there is no difference in the results between group 1 and group 2 . 
Table 2. Quality of reduction and incidence of complications after surgery with the modified Kocher-Langenbeck approach.

\begin{tabular}{|c|c|c|c|c|}
\hline & $\begin{array}{c}\text { Total } \\
\text { (n=13) }\end{array}$ & $\begin{array}{l}\text { Group } 1 \\
(n=7)\end{array}$ & $\begin{array}{l}\text { Group } 2 \\
(n=6)\end{array}$ & $P$ value ${ }^{\S}$ \\
\hline Anatomical $(\leq 2 \mathrm{~mm})$ & $13(100 \%)$ & $7(100 \%)$ & $6(100 \%)$ & \\
\hline \multicolumn{5}{|l|}{ Complications ( $\mathrm{n}, \%)$} \\
\hline Sciatic nerve injury & $0(0 \%)$ & $0(0 \%)$ & $0(0 \%)$ & 1.000 \\
\hline Superior gluteal neurovascular injury & $0(0 \%)$ & $0(0 \%)$ & $0(0 \%)$ & 1.000 \\
\hline Heterotopic ossification & $0(0 \%)$ & $0(0 \%)$ & $0(0 \%)$ & 0.165 \\
\hline Osteonecrosis of femoral head & $0(0 \%)$ & $0(0 \%)$ & $0(0 \%)$ & 1.000 \\
\hline Post-traumatic osteoarthritis & $2(15.4 \%)$ & $0(0 \%)$ & $2(33.3 \%)$ & 0.165 \\
\hline
\end{tabular}

Group1, transverse with or without posterior wall acetabular fracture; group 2, posterior wall acetabular fracture alone.

$\S P$ value, the calculated probability showing that there is no difference in the results between group 1 and group 2. 
Table 3. Clinical outcomes at 12 months after surgery with the modified Kocher-Langenbeck approach.

\begin{tabular}{|c|c|c|c|c|}
\hline & $\begin{array}{l}\text { Total } \\
\text { (n=12) }\end{array}$ & $\begin{array}{l}\text { Group } 1 \\
(n=7)\end{array}$ & $\begin{array}{l}\text { Group } 2 \\
(n=5)\end{array}$ & $P$ value $^{\S}$ \\
\hline Modified Harris hip score (mean, range) & $90.6(81-100)$ & $87.7(81-94)$ & $94.6(91-100)$ & $0.014^{*}$ \\
\hline
\end{tabular}

Group1, transverse with or without posterior wall acetabular fracture; group 2, posterior wall acetabular fracture alone.

$\S P$ value, the calculated probability to determine whether there is a difference in the results between group 1 and group 2.

* The difference is significant $(P<0.05)$. 
A

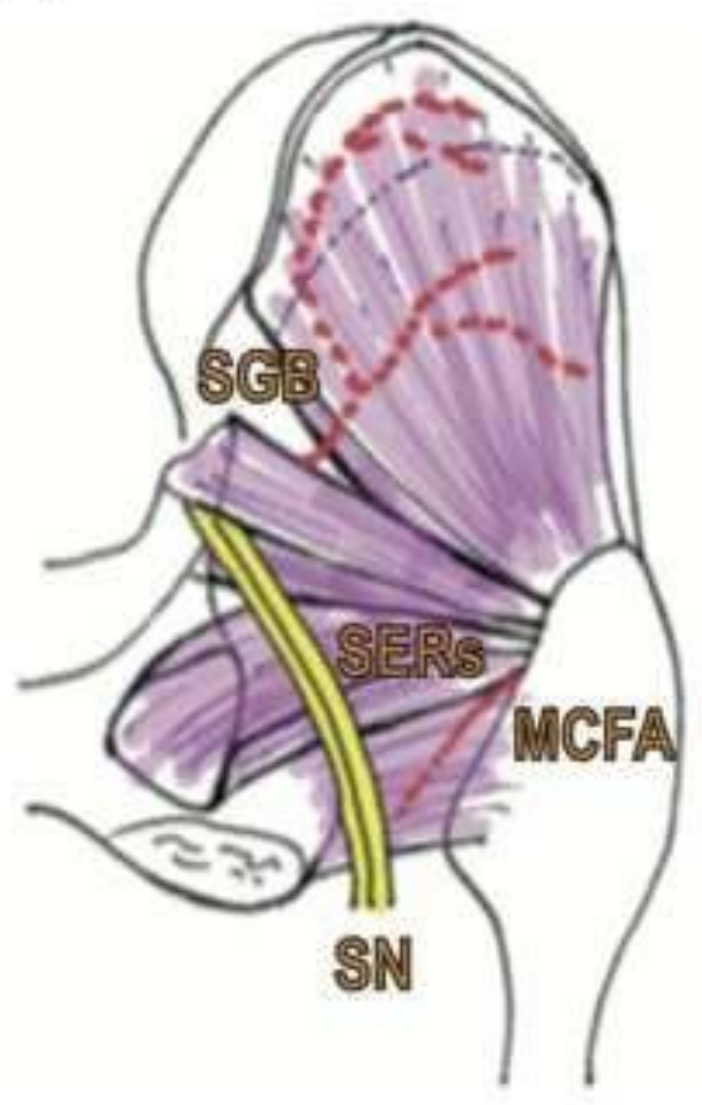

B

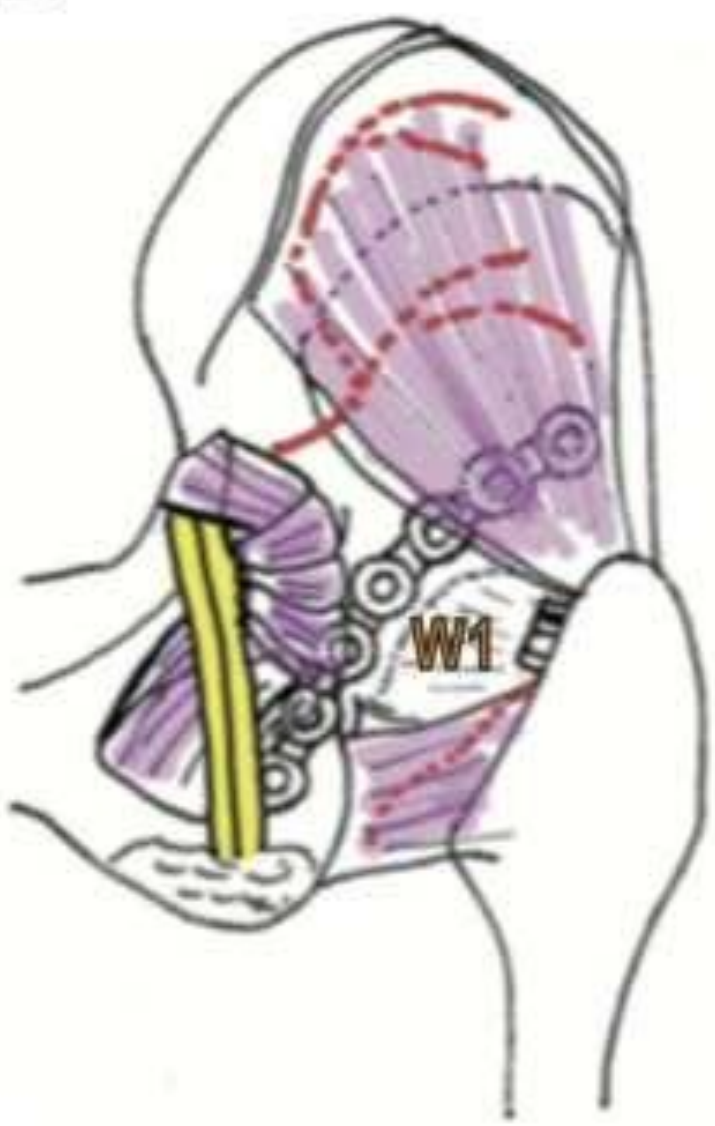

C

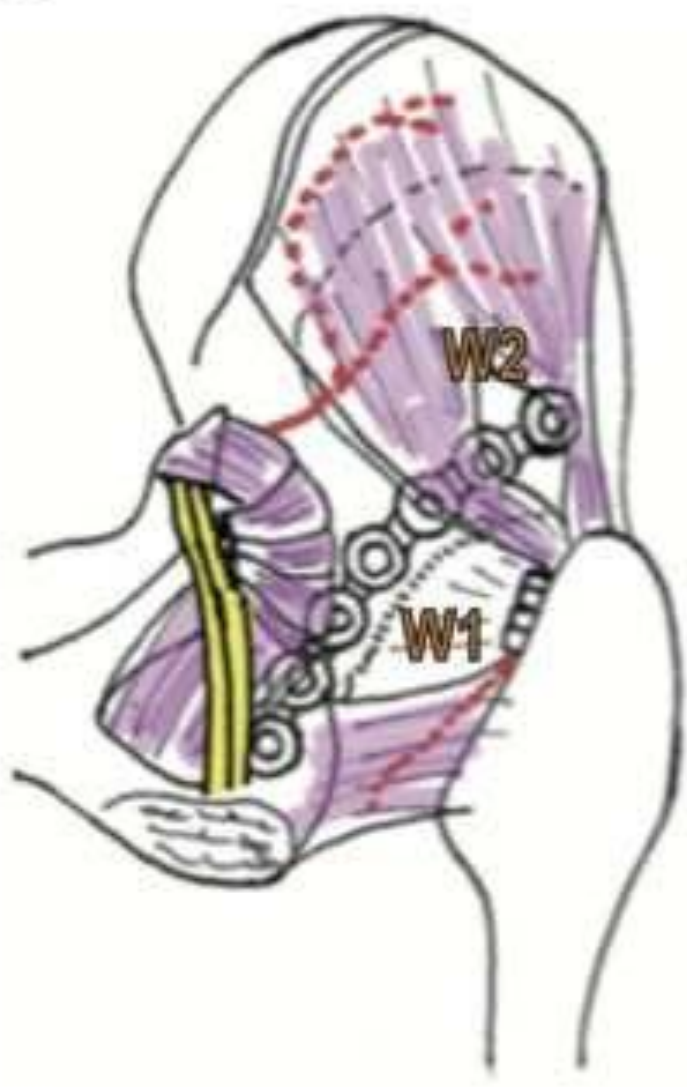



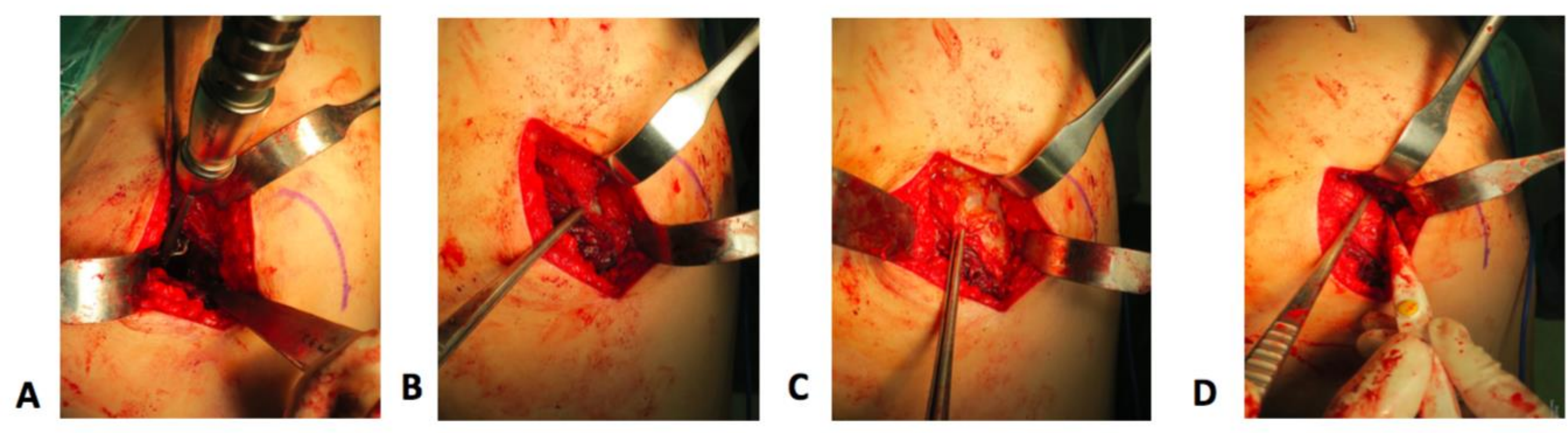

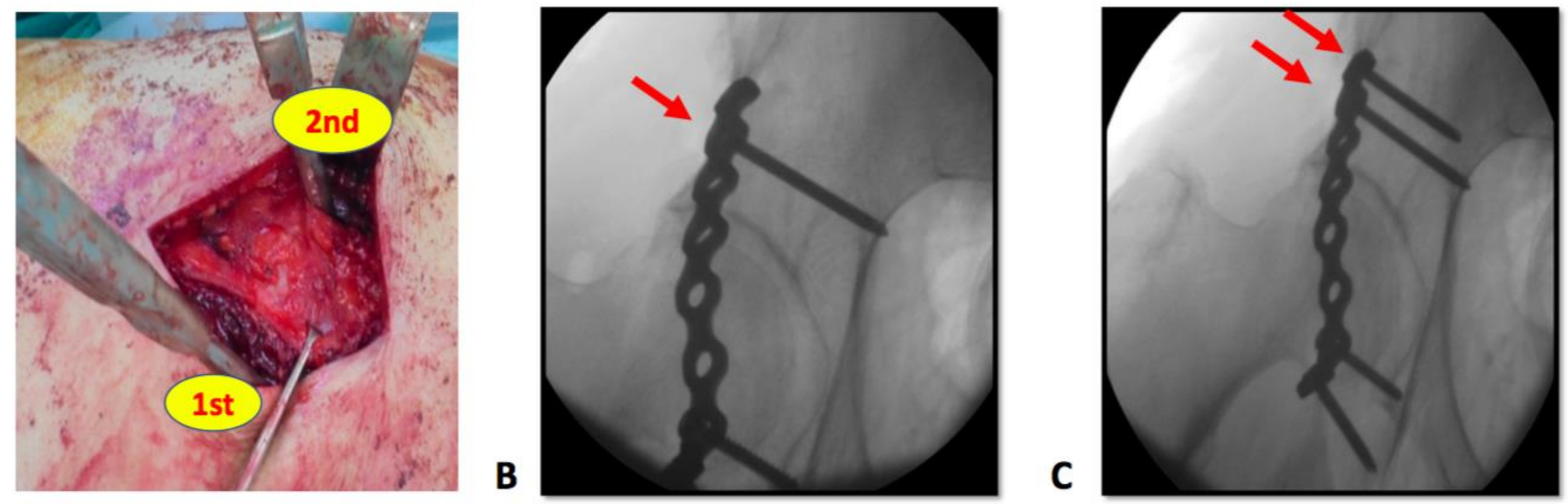

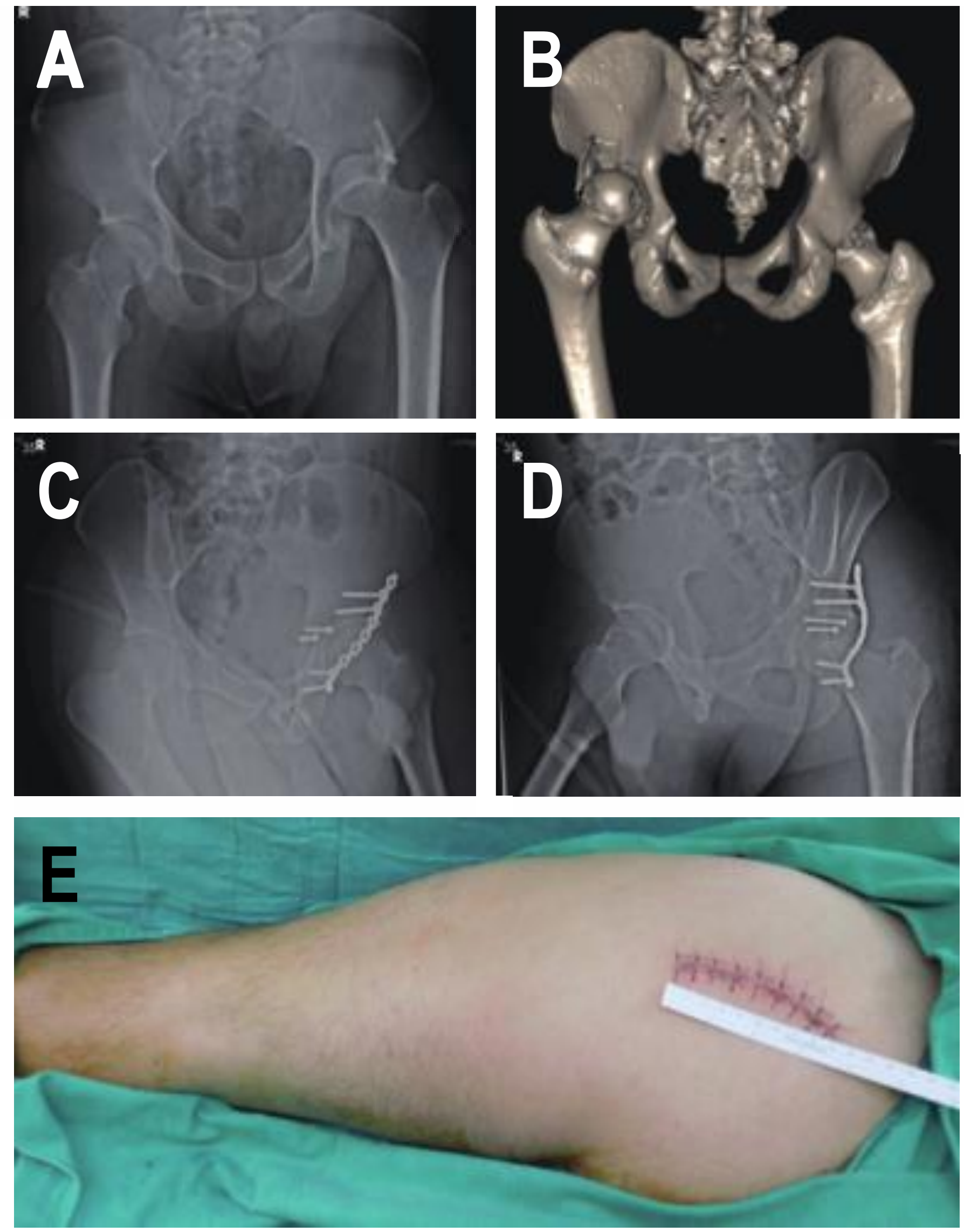


\section{Figures}

A

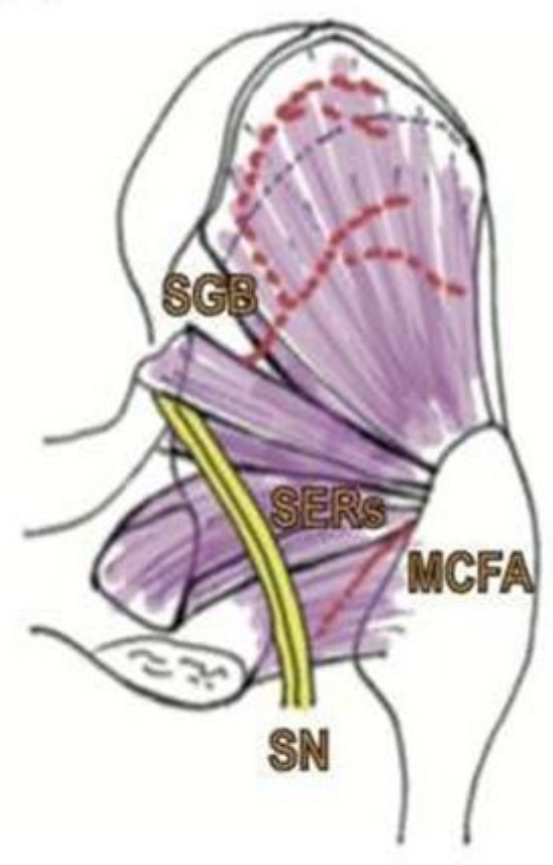

B

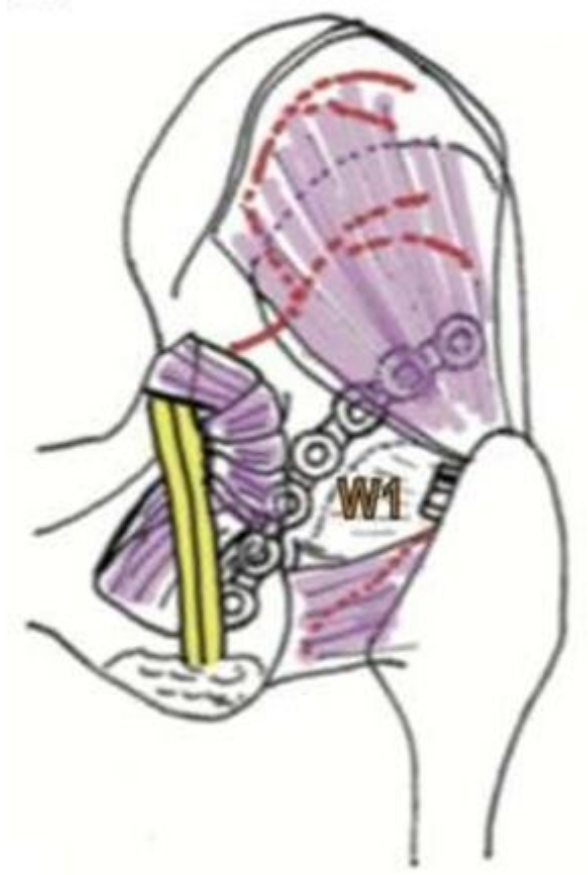

c

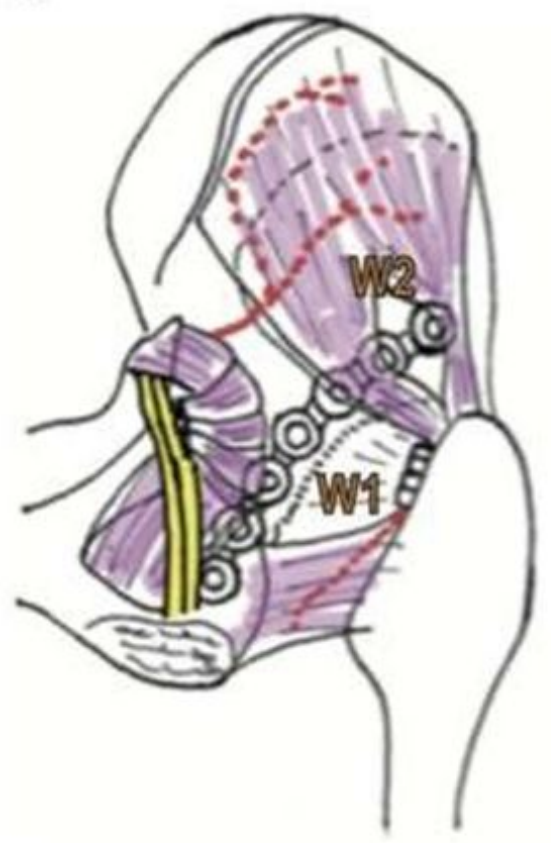

Figure 1

The modified "one-incision two-window" Kocher-Langenbeck surgical approach. (A) Posterior structures of the right hip joint. (B) Window 1 (W1) is used for controlling the posterior structures by gently elevating the short external rotators (SERs) as a flap, which preserves the medial circumflex femoral artery (MCFA) and protects the sciatic nerve (SN). After provisional reduction and fixation, a precontoured long pelvic reconstruction plate can be slid under the gluteal muscles, in line with the posterior rim of the acetabulum, spanning from the ischium to the superior wall area. Through this window, the distal end of the plate is fixed with two screws into its concave bend. (C) Window 2 (W2) is used for governing the superior wall area by splitting the gluteal muscles, which avoids vigorous retraction and prevents superior gluteal neurovascular bundle (SGB) injury. Through this window, the proximal plate screws can be applied accurately and safely in direct view. 

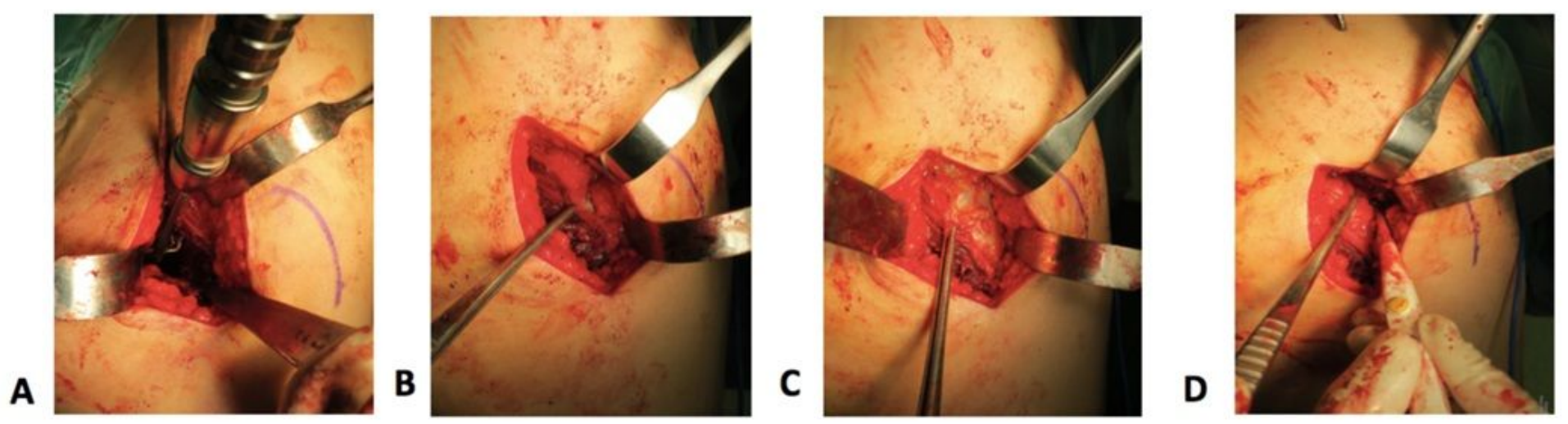

\section{Figure 2}

The procedure of making the second window. (A) Applying the distal screws at ischium through the first window. (B\&C) Anteriorly dissect the fascia lata upon the fascia layer. (D) Incise the fascia lata at the proximal screw level, then split the gluteal muscle with finger to the plate to make the second window.

A

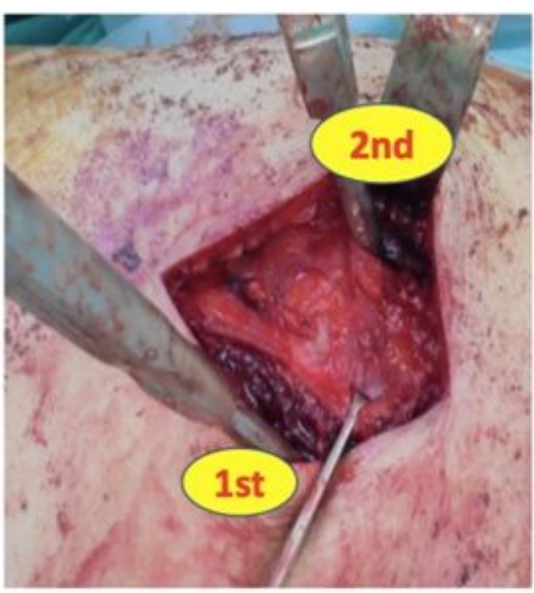

B

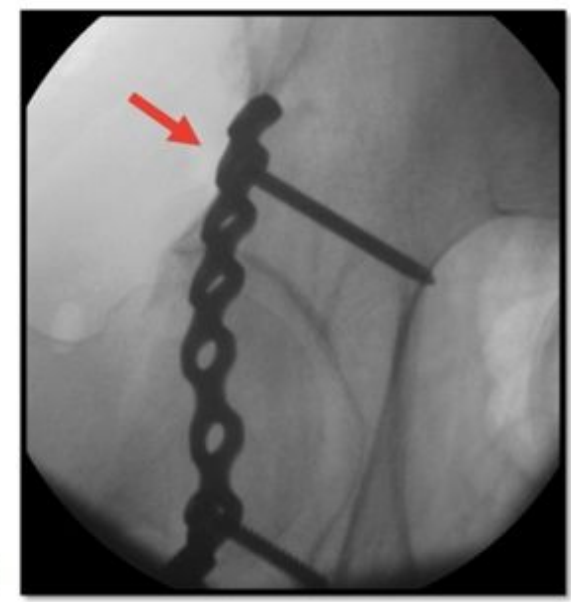

C

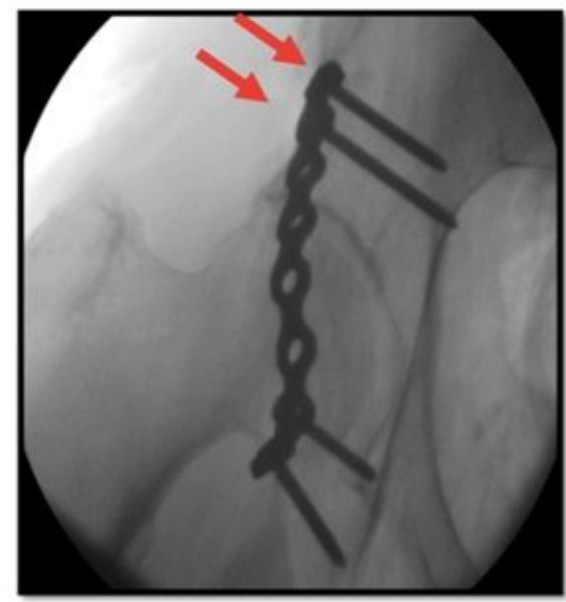

Figure 3

(A) represents the first and second windows. (B\&C) represent the proximal screws applying from the second window with verification by fluoroscopy.

\section{Supplementary Files}

This is a list of supplementary files associated with this preprint. Click to download.

- STROBEStatement.pdf

- SupplementalDigitalContent1.jpg 